\title{
Effect of an optical negative index thin film on optical bistability
}

\author{
N. M. Litchinitser \\ Department of Electrical Engineering and Computer Science, University of Michigan, 1301 Beal Avenue, \\ Ann Arbor, Michigan 48109, USA \\ I. R. Gabitov \\ Department of Mathematics, University of Arizona, 617 North Santa Rita Avenue, Tucson, Arizona 85721, USA \\ and Theoretical Division, Los Alamos National Laboratory, Los Alamos, New Mexico 87545, USA
}

A. I. Maimistov

Department of Solid State Physics, Moscow Engineering Physics Institute, Kashirskoe shosse 31, Moscow, Russian Federation 115409

V. M. Shalaev

School of Electrical and Computer Engineering and Birck Nanotechnology Center, Purdue University, West Lafayette, Indiana 47907, USA

Received June 29, 2006; revised September 24, 2006; accepted September 27, 2006; posted October 19, 2006 (Doc. ID 72511); published December 23, 2006

We investigate nonlinear transmission in a layered structure consisting of a slab of positive index material with Kerr-type nonlinearity and a subwavelength layer of linear negative index material (NIM) sandwiched between semi-infinite linear dielectrics. We find that a thin layer of NIM leads to significant changes in the hysteresis width when the nonlinear slab is illuminated at an angle near that of total internal reflection. Unidirectional diodelike transmission with enhanced operational range is demonstrated. These results may be useful for NIMs characterization and for designing novel NIMs-based devices. (C) 2006 Optical Society of America

OCIS codes: $190.1450,310.6860,160.4670$.

Recent experimental demonstrations of negative refractive index materials at optical frequencies ${ }^{1-4}$ open a fundamentally new branch of modern optics and photonics and new possibilities for manipulating light waves. Most of the theoretical studies of linear and nonlinear optical effects in negative index materials (NIMs) to date have focused on bulk NIM structures or periodic stacks of NIM and positive index material (PIM) layers. ${ }^{5-11}$ However, currently these materials are only available in the form of a single film with a thickness of about $150 \mathrm{~nm} .{ }^{1,2}$ In such a thin film, the negative refraction property reveals itself in a phase shift (phase advance) that was measured interferometrically in the experiments reported in Refs. 1 and 2.

In this Letter we investigate the effect of a thin linear layer of NIM on the transmission properties of a bilayer consisting of a layer of NIM and a nonlinear slab of conventional PIM. We propose to utilize the phenomenon of optical bistability for NIMs characterization and novel device applications.

Optical bistability is a class of optical phenomena in which a system can exhibit two steady transmission states for the same input intensity. ${ }^{12}$ The inputoutput characteristic of such a system forms a hysteresis loop. The structure under consideration consists of a semi-infinite linear cladding $(c l 1)$, nonlinear optically thick layer-layer $1(N L)$, linear NIM thin film-layer 2 (NIM), and a semi-infinite linear cladding (cl2) (Fig. 1). This structrure is illuminated at the angle near that of total internal reflection (TIR). We demonstrate that (i) an optically thin layer of NIM significantly modifies the bistable nonlinear transmission characteristics of this simple layered structure, and (ii) when the structure shown in Fig. 1 is illuminated leftward versus rightward, unidirectional transmission with increased operational range can be achieved.

The nonlinear layer is characterized by a dielectric permittivity $\varepsilon=\varepsilon^{L}+\varepsilon^{N L}\left(|E|^{2}\right)$, where $\varepsilon^{L}$ is the linear part of the relative dielectric permittivity, which generally can be complex, $\varepsilon^{N L}\left(|E|^{2}\right)=\varepsilon^{L} \varepsilon_{0} c n_{2}|E|^{2}$ is the nonlinear, intensity dependent part of the dielectric permittivity, $n_{2}$ is the nonlinear refractive index.

In the nonlinear layer, the following wave equation is solved for TE polarization

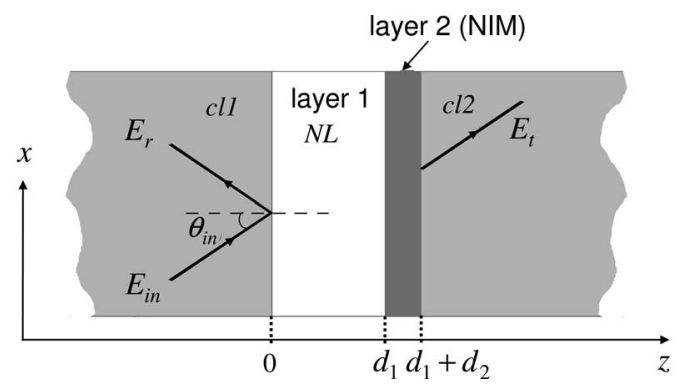

Fig. 1. Schematic of the layered structure under investigation. $E_{\text {in }}$ is the incident field, $E_{r}$ is the reflected field, and $E_{t}$ is the transmitted field. 

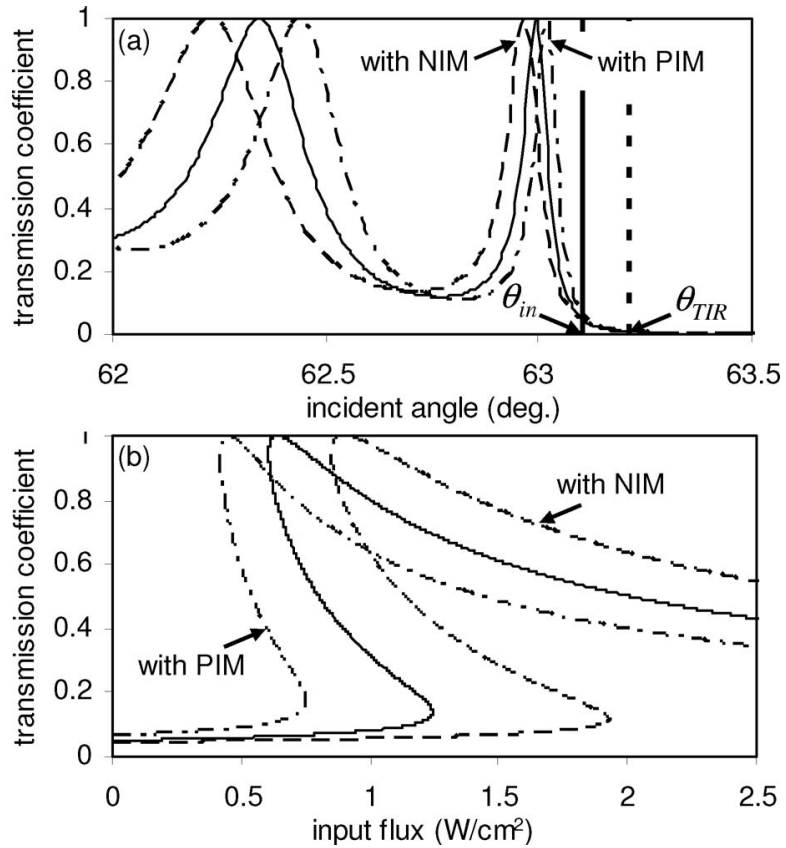

Fig. 2. (a) Linear transmission coefficient versus the angle of incidence for a single layer 1 (solid curve), the transmission coefficient for a combination of layer 1 and a NIM thin film (dashed curve), and the transmission coefficient for a combination of layer 1 and a PIM thin film (dotted-dashed curve). (b) Transmission coefficient versus the input flux corresponding to the three configurations in (a).

$$
\frac{\mathrm{d}^{2} E}{\mathrm{~d} \zeta^{2}}+p^{2} E+\mu^{L} \varepsilon^{N L}\left(|E|^{2}\right) E=0
$$

where $\zeta=k z, k=\omega / c$ is the wave vector, and $p^{2}=\varepsilon^{L} \mu^{L}$ $-\beta^{2}, \beta=\left(\varepsilon_{c l 1} \mu_{c l 1}\right)^{1 / 2} \sin \theta_{i n}, \theta_{i n}$ is the angle of incidence, $\mu^{L}$ is the linear part of the relative magnetic permeability. Here it is assumed that the magnetic response is linear in all layers.

As a first step, we consider the angular dependence of the transmission coefficient for the three cases: (i) nonlinear optically thick slab with $\varepsilon_{1}^{L}=2.46, \mu_{1}^{L}=1$, $d_{1}=2.5 \mu \mathrm{m}$, and $n_{2}=2 \times 10^{-9} \mathrm{~m}^{2} / \mathrm{W}$ surrounded by a linear dielectric with a higher dielectric constant; (ii) the same slab as in case (i) but combined with a thin NIM layer characterized by $\varepsilon_{2}^{L}=-2.46, \mu_{2}^{L}=-1, d_{2}$ $=150 \mathrm{~nm}$; and (iii) the same slab combined with a thin PIM layer characterized by $\varepsilon_{2}^{L}=2.46, \mu_{2}^{L}=1, d_{2}$ $=150 \mathrm{~nm}$. In all cases, claddings are made of linear dielectric with $\varepsilon_{c l 1}^{L}=\varepsilon_{c l 2}^{L}=3.087, \mu_{c l 1}^{L}=\mu_{c l 2}^{L}=1$. Figure 2(a) shows the angular dependences of the linear transmission coefficient for cases (i)-(iii). The vertical dashed line corresponds to the TIR angle $\theta_{T I R}$ $=63.21^{\circ}$ and the vertical solid line corresponds to the incident angle $\theta_{\text {in }}=63.1^{\circ}$ used in all simulations in this Letter. Figure 2(a) shows that in the presence of the NIM (PIM) thin film, transmission maxima in the linear transmission spectrum shift to smaller (larger) angles with respect to the case of a single nonlinear layer. The corresponding transmission coefficient as a function of the input flux is shown in Fig. 2(b).

The layered structure in Fig. 1 can be viewed as an analog of a resonator filled with intensity-dependent material. A linear NIM (PIM) thin film placed between the nonlinear slab and the second cladding (cl2) introduces an additional phase shift that affects the resonant condition leading to the transmission spectrum shift shown in Fig. 2(a). It is noteworthy that although the NIM (PIM) film is very thin, the effect of it on the nonlinear optical response of the entire structure turns out to be very significant. As discussed above, negative refraction reveals itself in a phase advance or a negative phase shift. Therefore, in the case of a NIM thin layer, the "resonator" length decreases, implying that the intensity-dependent nonlinear index change required for switching the system to the high-transmission state should increase. Therefore, we predict that the bistability threshold should increase in the case of the NIM, while the opposite effect should occur in the case of the PIM. Numerical results shown in Fig. 2(b) confirm this prediction.

Next, we study the effects of the NIM film parameters on the hysteresis curve and compare the results to those for the PIM case. Figure 3(a) shows the transmission coefficient versus input flux for the fixed dielectric permittivity $\varepsilon_{2}^{L}=-0.5$ and varying magnetic permeability $\mu_{2}^{L}$ compared to those for PIM thin film with the same absolute values of $\varepsilon_{2}^{L}$ and $\mu_{2}^{L}$. The hysteresis width increases in the case of NIM film, as the absolute value of $\mu_{2}^{L}$ increases. In the PIM case, hysteresis width decreases as $\mu_{2}^{L}$. The bistability threshold increases also in the NIM case, in agreement with the predictions of the simple resonator analogy based model discussed above. Finally, we investigate the effect of varying both $\varepsilon_{2}^{L}$ and $\mu_{2}^{L}$ while the refractive index is fixed at $n=-1.5$ [Fig. 3(c)] and $n=1.5$ [Fig. 3(d)]. Again, we find that the hysteresis curves' widths and hysteresis thresholds are significantly increased in the case of NIM, while an opposite effect is observed in the case of PIM. We note that in all the examples considered here, NIMs were assumed to be lossless. The detailed study of the effect of loss in NIMs on the hysteresis depth and width will be addressed elsewhere.

Up to this point, we assumed that the light always impinges the structure in Fig. 1 from the left. We now examine reciprocity in the bilayer structure comprising a NIM film. Figure 4 shows hysteresis curves for the cases when the structure shown in Fig. 1 is illuminated leftward versus rightward for $\left|\varepsilon_{2}^{L}\right|=1,\left|\mu_{2}^{L}\right|$ $=2$. In Fig. 4, solid curves correspond to the NIM case when the structure is illuminated from left to right [curve (1)] and from right to left [curve (2)]; dashed curves correspond to the PIM case when the structure is illuminated from left to right [curve (3)] and from right to left [curve (4)]. We find that the range of intensities corresponding to unidirectional transmission is significantly increased in the case of a bilayer comprising a thin film of NIM.

In summary, we studied numerically the nonlinear transmission properties of a layered structure consisting of a nonlinear slab and a linear thin layer of NIM. We found that even a very thin (subwavelength) film of NIM significantly modifies the nonlin- 

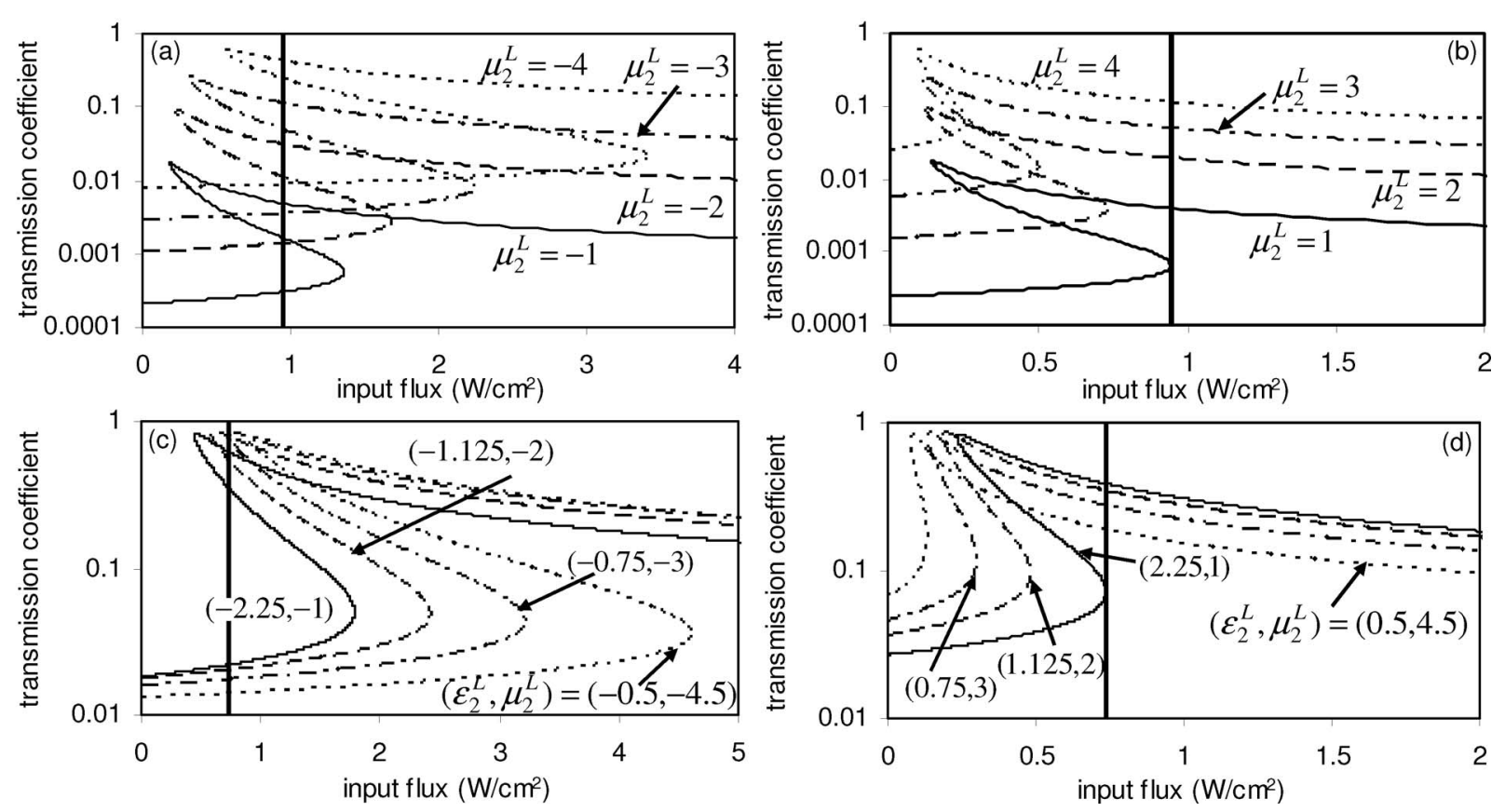

Fig. 3. Comparison of the transmission coefficient versus the input flux for the case of fixed dielectric permittivity in the (a) NIM and (b) PIM layers and varying magnetic permeability, and for the case of fixed refractive index in the (c) NIM and (d) PIM layers and varying both magnetic permeability and dielectric permittivity. Vertical straight lines indicate the maximum bistability threshold in the PIM case.

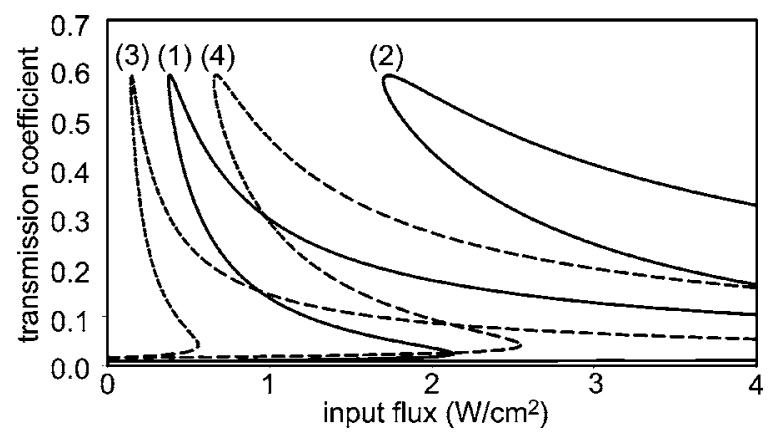

Fig. 4. Transmission coefficient versus the input flux for the layered structure shown in Fig. 1 when the light enters from the left [curve (1)] and when the light enters from the right [curve (2)]. Curves (3) and (4) correspond to the case of the PIM thin layer.

ear response of this structure. It is noteworthy that although we have considered NIM with $\varepsilon$ and $\mu$ being simultaneously negative, the effects described here rely on the refractive index itself being negative and, thus, we expect the results to be valid for other types of NIMs. ${ }^{13}$ In addition, we examined the nonlinear optical response as a function of directionality of the incident light and found a significantly increased (compared to the PIM case) range of input intensities corresponding to the unidirectional transmission. These results may be useful for the characterization of NIMs, as well as for novel device applications such as optical memory and optical diode.

The authors are grateful to Askhat Basharov and Vladimir Drachev for enlightening discussions. This work was supported in part by National Science Foundation (NSF) Nanoscale Interdisciplinary Research Teams award ECS-0210445; U.S. Army Re- search Office (ARO) grant W911NF-04-1-0350; Multidisciplinary University Research Initiative award 50342-PM-MUR; NSF award DMS-050989; U.S. Department of Energy (DOE) contract DE-AC5206NA25396; and the DOE Office of Science Advanced Scientific Computing Research Program in Applied Mathematics Research, Proposition 301 State of Arizona. N. Litchinitser's e-mail address is natashan@eecs.umich.edu.

\section{References}

1. V. M. Shalaev, W. Cai, U. K. Chettiar, H. Yuan, A. K. Sarychev, V. P. Drachev, and A. V. Kildishev, Opt. Lett. 30, 3356 (2005).

2. S. Zhang, W. Fan, C. Panoiu, K. J. Malloy, R. M. Osgood, and S. R. Brueck, Phys. Rev. Lett. 95, 137404 (2005).

3. A. N. Grigorenko, A. K. Geim, H. F. Gleeson, Y. Zhang, A. A. Firsov, I. Y. Khrushchev, and J. Petrovic, Nature 438, 335 (2005).

4. G. Dolling, C. Enkrich, M. Wegener, C. M. Soukoulis, and S. Linden, Opt. Lett. 31, 1800 (2006).

5. V. M. Agranovich, Y. R. Shen, R. H. Baughman, and A. A. Zakhidov, Phys. Rev. B 69, 165112 (2004).

6. G. D'Aguanno, N. Mattiucci, M. Scalora, and M. J. Bloemer, Phys. Rev. Lett. 93, 213902 (2004).

7. A. A. Zharov, N. A. Zharova, I. V. Shadrivov, and Yu. S. Kivshar, Appl. Phys. Lett. 87, 091104 (2005).

8. M. W. Feise, I. V. Shadrivov, and Yu. S. Kivshar, Phys. Rev. E 71, 037602 (2005).

9. R. S. Hegde and H. Winful, Opt. Lett. 30, 1852 (2005).

10. A. K. Popov and V. M. Shalaev, Opt. Lett. 31, 2169 (2006).

11. A. K. Popov and V. M. Shalaev, Appl. Phys. B 84, 131 (2006).

12. H. M. Gibbs, Optical Bistability (Academic, 1985).

13. A. L. Efros and A. L. Pokrovsky, Solid State Commun. 129, 643 (2004). 